\title{
The minimal perimeter for $N$ confined deformable bubbles of equal area
}

\author{
S.J. Cox and E. Flikkema \\ Institute of Mathematics and Physics, \\ Aberystwyth University, Ceredigion SY23 3BZ, UK.
}

Submitted: Feb 3, 2010; Accepted: Mar 11, 2010; Published: Mar 22, 2010

Mathematics Subject Classifications: 05B40, 52B05, 74G65

\begin{abstract}
Candidates to the least perimeter partition of various polygonal shapes into $N$ planar connected equal-area regions are calculated for $N \leqslant 42$, compared to partitions of the disc, and discussed in the context of the energetic groundstate of a two-dimensional monodisperse foam. The total perimeter and the number of peripheral regions are presented, and the patterns classified according to the number and position of the topological defects, that is non-hexagonal regions (bubbles). The optimal partitions of an equilateral triangle are found to follow a pattern based on the position of no more than one defect pair, and this pattern is repeated for many of the candidate partitions of a hexagon. Partitions of a square and a pentagon show greater disorder.

Candidates to the least perimeter partition of the surface of the sphere into $N$ connected equal-area regions are also calculated. For small $N$ these can be related to simple polyhedra and for $N \geqslant 14$ they consist of 12 pentagons and $N-12$ hexagons.
\end{abstract}

\section{Introduction}

A dry foam is a collection of polyhedral bubbles surrounded by thin films. Its highinterface structure leads to a multitude of industrial and domestic uses [1]. The surface energy of a three-dimensional foam is the surface area of the films multiplied by the surface tension of each one [1]. A foam in equilibrium attains a local minimum of this energy, subject to the constraint of fixed bubble volumes. In doing so, it satisfies Plateau's rules $[2,3]$ : three and only three films meet in a line (a Plateau border) at $120^{\circ}$ and these lines meet with four-fold tetrahedral symmetry. In addition, the Laplace Law relating pressure difference and curvatures gives the further condition that each film is a surface of constant mean curvature.

A celebrated problem in this context is due to Kelvin [4, 5]: what is the least energy partition of space into cells of equal volume? Kelvin's solution, a tetrakaidecahedral 
bubble in the form of a truncated octahedron with six quadrilateral and eight hexagonal faces, still stands as the best monohedral tiling ever found. A proof of its optimality is, however, elusive. One hundred years after Kelvin considered the problem, Weaire and Phelan [6] discovered a partition of space with lower energy; the Weaire-Phelan structure has a unit cell consisting of eight bubbles of two different topologies.

Given the complexity of the search for a global minimum in the Kelvin problem, it makes sense to retreat to two dimensions. Consider for example, a foam squeezed between parallel plates, so that the films form a network of lines surrounding the bubbles. The surface energy of this two-dimensional foam is simply the total perimeter of the lines (or edges) multiplied by surface tension. The rules of equilibrium now imply that each edge is a circular arc and that they meet in threes at angles of $120^{\circ}$. The bubble areas are considered fixed and we seek the arrangement of bubbles that gives the global minimum of perimeter. For bubbles filling the plane, the hexagonal honeycomb has been proved to be optimal in this sense [7]. This result was widely believed but difficult to prove, as is the case for so many of these minimal perimeter problems. The approach adopted here, and expanded upon below, is to seek candidates to such problems numerically and, except in some special cases, forgo any attempt at a proof that the structure is a global minimum. Instead, we rely on a procedure that calculates the perimeter of many different candidates, giving a high probability but no guarantee that we will find the "best" one.

So, the honeycomb conjecture is proven, but there are many variations on the conjecture that are worthy of further exploration. One obvious direction is to relax the condition that all bubbles have equal area $[8, \S 15.9][9]$. Another is to consider finite collections of $N$ equal-area bubbles (clusters), which we do here. An important assumption is that in the global minimum each bubble consists of a single component. We retain this assumption of connectedness for all problems considered here. The difficulty of proving this assumption is the major stumbling block to proving optimality in general.

For small numbers of bubbles, $N \leqslant 3$, there are various proofs of optimality for free clusters (in 2D, 3D and beyond); see e.g. [8, 10, 11, 12, 13]. For example, a single bubble $(N=1)$ forms a circle, and two bubbles form what is known as the standard double bubble - see the last column of figure 1. Cox et al. [14] "shuffled" a monodisperse cluster of bubbles from one configuration to another by performing neighbour-switching T1 changes [15] on an initially defect-free cluster to explore the space of possible candidates. Those results for the least-perimeter arrangement of $N$ monodisperse bubbles, along with a number of more recent improvements [16], are shown in figures 1 to 5 .

The colour scheme in the figures requires explanation: topological defects are classified using the idea of charge [17]. Bulk bubbles have a charge $q=6-n$, where $n$ is the number of sides. Thus hexagons have zero charge and are not coloured. In the same way, peripheral bubbles have charge $q=5-n$. The total charge of the cluster is then $\sum q=6$. Cox et al. [14] found that in the free cluster case, positive (coloured red in the figures if $q=1$, if $q \geqslant 2$ ) and negative (yellow) charges tend to be associated, and that the remaining positive charges are usually well-spaced around the periphery of the cluster. As the size of the cluster grows, the result of the honeycomb conjecture suggests that bubbles far from the periphery of the cluster are likely to be hexagonal, as is indeed the case. 
Simulations by Cox and Graner [18] showed that for $N$ up to 10,000 the trade-off between reducing the length of the periphery of the cluster and attaining regular hexagons in the bulk is "won" by the bulk. That is, the perimeter of the cluster is minimized by a cluster that has a periphery that is itself hexagonal, allowing the bulk to consist only of regular hexagons, rather than rounding the cluster to reduce the length of the periphery. This suggestion has not gained universal agreement: Morgan [19], for example, expects the periphery of the optimal cluster to become circular at even higher $N$.

Bounds on the perimeter $E$ of a finite cluster of hexagons have been given by Heppes and Morgan [20]. These are asymptotically of the form

$$
E_{f}(N) / L=3 N+k \sqrt{N}
$$

for some parameter $k$. The first term is the contribution of hexagonal bubbles in the bulk and the $\sqrt{N}$ term is a correction due to the peripheral bubbles. Thus $L$ is the length of one edge of a regular hexagonal bubble (since each edge is shared between two bubbles) of area $A=\frac{1}{2} 3 \sqrt{3} L^{2}$. In fitting this form to the results of simulations, the second term must also account for deviations of hexagons from regularity and any non-hexagonal bubbles in the centre, or bulk, of the cluster. Cox et al. [14] found $k=3.068$ for free clusters.

A further refinement of the problem of finding the least perimeter arrangement of bubbles is to consider a number of bubbles confined within a given boundary. The equilibrium conditions are augmented by the rule that edges meet the bounding walls at $90^{\circ}$. Cañete and Ritore [21] proved that the best arrangement of three bubbles within a circle is the same topology as in the free cluster for $N=3$ [13]. A number of authors have made conjectures in the monodisperse case for $N$ up to $6[21,22,23,24]$ and Cox [16] performed a search for $N$ up to 45 that confirmed most of these (see the fifth column of figures 1 to 5, which also contains improvements for $N=40$ (this work) and $N=42$ [29] to those given in [16]).

For different confining geometries, there are only conjectures $[22,23,24]$ for the square and equilateral triangle, again for $N$ up to 6 . Here, we consider partitions of both of these, as well as a pentagonal and hexagonal boundary, for $N$ up to 42 . For each boundary shape we seek the least perimeter partition into $N$ bubbles of equal area, equivalent to the energetic groundstate for $N$ monodisperse bubbles or the optimal packing of equalarea objects. We examine values of $N$ up to 42 and record the least perimeter, the number of peripheral bubbles $N_{p}$ and the topology (number of sides) of the bubbles in the bulk of the cluster. That is, we determine candidates to the least perimeter arrangement, and examine the influence of the periphery in creating deviations of the cluster from the regular hexagonal lattice.

In addition, we consider the least perimeter partition of the surface of the unit sphere into $N$ regions of equal area. In this case each edge is an arc of a great circle, and the edges meet in threes as before. The case $N=2$ is solved by two hemispherical shells. There are proofs that three identical strips joining the poles is optimal for $N=3$ [25], and that the optimal partition into $N=4$ regions consists of four equal triangles [26]. Hales [27] proved that for $N=12$ the optimal partition is the one based on the pentagonal dodecahedron. It is believed that the cube provides the topology of optimal partition into 
$N=6$ regions. Indeed, we find that the ten geodesic partitions of the sphere that are three-connected at $120^{\circ}$, which occur for $N=2-10,12$ (see [28]), provide the minimum perimeter. Further conjectures can be found in [30, 31].

Our aim is partly to inspire the derivation of exact results. In addition, in the same way that the optimal arrangement of a free cluster of bubbles appears to predict the arrangement of retinal cells in Drosophila [32], the solutions found here may provide information about other biological structures, such as the arrangement of seeds in a flower $[33,34]$. They are also reminiscent of the results of more traditional packing problems, in which objects should fill a given space with least deformation.

\section{Method}

The value of surface tension, which is equal for all edges, is taken as one.

For small $N \leqslant 6$, all possible combinatorial types can usually be enumerated, which provides a check on the numerical procedures described below. For certain boundary shapes, there are magic numbers of bubbles: configurations in which the least perimeter solution has no topological defects. Two examples are (i) hexagonal numbers, of the form $3 i^{2}-3 i+1$ for $i=1,2, \ldots$, which were found to be optimal for free clusters, those confined in a circle, and, as we will see below, are optimal for those confined in a hexagon; (ii) triangular numbers, of the form $N_{T}=\frac{1}{2} i(i+1)$, which we find (see below) to be optimal for bubbles confined in an equilateral triangle; that is, for values of $N=N_{T}$ the cluster

formed by cutting a triangular segment from a hexagonal honeycomb and allowing it to deform slightly to satisfy area constraints provides the global minimum.

\subsection{Polygonal boundary}

Candidates to the optimal arrangement of bubbles within a polygonal boundary are created as follows (the method is similar to the one described in [16]): $N$ points, which represent bubble centres, are scattered at random in a unit square. The points are moved so as to minimize a potential (see below) and then a Voronoi partition of these $N$ seed points is calculated [35], The resulting structure is imported into the Surface Evolver [36], where each Voronoi cell represents a bubble. The polygonal boundary is set to have sides of unit length, the bubble areas are prescribed to be equal and we then use Evolver's circular arc mode to converge to a minimum of the perimeter. The equilibrium perimeter $E$ of the candidate configuration is then calculated, including the length of the boundary to facilitate comparisons between different boundary shapes.

This complete procedure is repeated for each local minimum of the potential, of which there are of the order of ten to twenty for each $N$, and the least-perimeter candidate recorded in each case. Following the initial perimeter minimization step, short edges are sequentially removed, through T1 neighbour switching processes, and the perimeter again minimized to seek better nearby minima.

We supplement this automated procedure with manipulations of the existing candidates by hand, based upon intuition about the structure and symmetry of the candidates, 
and any patterns noticed.

There is no reason why any given inter-particle potential should provide good candidates to the minimum perimeter problem. Indeed, there are many well-known potentials [37], the solutions to which may show a precise correspondence between the particle positions and the arrangement of bubble centres in the optimal partition, at least at small $N$. We therefore choose a number of different potentials and compare their effectiveness in determining the least perimeter candidate.

Each potential consists of a sum of different contributions:

$$
V=c_{1} \sum_{i} \vec{r}_{i}^{2}+\sum_{i} \sum_{j \neq i} V_{i j}
$$

where $\vec{r}_{i}=\left(x_{i}, y_{i}\right)$ is the position vector of the $i^{\text {th }}$ particle. The first term is a symmetric harmonic confining potential that keeps the particles close to the origin, weighted by a constant $c_{1}$, usually equal to one. The second term is the inter-particle potential, which tries to keep the points well-spaced. We tried three possibilities for $V_{i j}$ :

$$
\begin{aligned}
V_{i j}^{\text {Coulomb }} & =\frac{1}{\left|\vec{r}_{i}-\vec{r}_{j}\right|} \\
V_{i j}^{\text {Squared }} & =\frac{1}{\left|\vec{r}_{i}-\vec{r}_{j}\right|^{2}} \\
V_{i j}^{\log } & =-\log \left|\vec{r}_{i}-\vec{r}_{j}\right| .
\end{aligned}
$$

In the triangular $(s=3)$ and square $(s=4)$ cases, we used only the second, squared, inter-particle potential in the second term, and added a further term that acts to make the boundary of the cluster of points polygonal:

$$
V_{\text {poly }}=c_{2} \sum_{i} \vec{r}_{i}^{2} \cos ^{2}\left(\theta_{i} \bmod (2 \pi / s)\right)
$$

with $\tan \theta_{i}=y_{i} / x_{i}$. The constant $c_{2}$ is between zero and one, and in these cases $c_{1}$ is reduced.

\subsection{The surface of a sphere}

To find candidates to the least perimeter partition of the surface of a sphere, the method is simpler. We choose the sphere to have radius $R=1$, centred at the origin, and tile it with $N$ bubbles of area $A=4 \pi R^{2} / N$. We use the Surface Evolver [36] in a "spherical arc mode", that represents each edge as an arc of a great circle, to minimize the total perimeter $P$.

We commence by covering the sphere with curvilinear triangles that have their base on the equator and their apices at one of the poles. By sequentially allowing neighbour switching topological changes on short edges and converging to a local equilibrium after each one, the perimeter of the pattern is reduced. We continue this process until the perimeter ceases to decrease, and then introduce further topological changes at random 
to search the nearby energy "landscape". We record the perimeter $E$ and the pattern of the topology with the least perimeter. A two-dimensional image of each pattern is obtained by projecting the vertices and edges on the surface of the sphere to the plane according to a Gauss map:

$$
\begin{aligned}
x^{\prime} & =\left(\frac{1}{2} \pi+\tan ^{-1}\left(\frac{z}{\sqrt{x^{2}+y^{2}}}\right)\right) \cos \left(\tan ^{-1}\left(\frac{y}{x}\right)\right) \\
y^{\prime} & =\left(\frac{1}{2} \pi+\tan ^{-1}\left(\frac{z}{\sqrt{x^{2}+y^{2}}}\right)\right) \sin \left(\tan ^{-1}\left(\frac{y}{x}\right)\right) \\
z^{\prime} & =-1 .
\end{aligned}
$$

It became apparent (see $\S 4$ ) that many candidates, particularly at larger $N$, consisted of arrangements of hexagons and pentagons only. We therefore implemented an additional procedure: the software CaGe [38] was used to enumerate all tilings of the sphere by hexagons and pentagons for each $N$. Each of these was imported into the Surface Evolver and its equilibrium perimeter found. This showed that the random search procedure described above was in general only finding optimal candidates for $N \leqslant 20$.

\section{Results}

\subsection{Triangular boundary}

Even in the monodisperse case, the least perimeter partition of an arbitrary triangle will depend on its precise shape. For example, in the case $N=3$ in an isosceles triangle there are three optimal arrangements, each of which is best for a certain range of angles [39]. We thus concentrate our effort on the equilateral case.

Our candidate configurations are shown in figures 1 to 5 , and the perimeters plotted in figure 6 and tabulated in Table 1 . We find the same candidates for $N=1,2,3$ and 6 as those found by Bleicher [23] and give better ones for $N=4$ and $N=5$. To the best of our knowledge, no candidates have been given for higher $N$. It can be shown, by enumerating all topologies, that the least-perimeter solution for $N=4$ does not have three-fold rotational symmetry [40] but is instead of the same form as the free cluster.

In the candidate configurations it is never optimal to have an edge emanating from an apex of the triangle. A semi-rigorous proof can be obtained by considering the partition of a scalene triangle into two bubbles.

For the triangular values, $N_{T}=1,3,6,10, \ldots, \frac{1}{2} i(i+1)$, the optimal candidate consists of part of a hexagonal honeycomb, albeit a deformed one that accommodates the area constraints. Based upon the number of peripheral bubbles in this defect-free case, we propose the following formula for $N_{p}$ :

$$
N_{p}=\left[\frac{3}{2}(\sqrt{1+8 N}-3)\right]
$$


where [] denotes the nearest integer. This expression fits the data well, except (at higher $N$ ) on either side of the triangular values where $N_{p}$ changes more steeply.

An approximate formula for the perimeter can be derived, based upon the idea that each bubble in the bulk attains its optimal hexagonal shape and that each wall bubble (except for those in the corners, which are not significant in this approximation) attains the optimal stretched-half hexagon shape that accommodates the area constraint. There are $3 N-2 N_{p}-3$ edges of length $L$ in the bulk and $N_{p}$ edges of length $5 L / 4$ that touch the walls, with $N_{p}$ given by (8). For a polygonal boundary with $s$ sides, we must add

$s$ walls of length $\bar{L}(s) / L=\sqrt{6 \sqrt{3} \tan (\pi / s) N / s}$. The resulting expression for the total perimeter is

$$
\begin{aligned}
E_{\text {approx }} & =\left(3 N-2 N_{p}-3\right) L+\frac{5}{4} N_{p} L+s \bar{L}, \\
\text { i.e. } E_{\text {approx }} / L & =3\left(N-\frac{1}{4} N_{p}-1\right)+\sqrt{6 \sqrt{3} \tan (\pi / s) N s} .
\end{aligned}
$$

with $s=3$ in this case.

Based upon the perimeter for the $N=3$ case, the expression $E / L=\left(3+N_{p} / \sqrt{12}\right) \sqrt{6 N}$ appears to give the perimeter for all triangular numbers, and thus provides a good lower bound for the perimeter for all $N$. A fit to the form in (1), $E_{f}(N) / L=3 N+k \sqrt{N}$ gives $k=4.302$.

For all other $N$ there is exactly one pair of defects, i.e. one bubble with positive topological charge adjacent to one bubble with negative topological charge. This provides a clear realization of the conjecture of Cox et al. [14] that defects should associate.

The position of the defect pair depends upon the proximity of $N$ to a triangular number. If $N$ is of the form $N_{T} \pm 1$, then the defect pair will touch the boundary. If $N$ is of the form $N_{T}+1$ then the positive charge will be against the boundary while if $N$ is of the form $N_{T}-1$, the negative charge will touch the boundary. If $N$ is two or more away from $N_{T}$, the defect pair will move into the bulk, keeping the same orientation of the positive-negative charge.

This suggests a recipe to generate the optimal candidate for each value of $N$, given the optimal defect-free candidate for the nearest $N_{T}$, as follows. Find the closest value of $N_{T}$ to $N$, and if there are two, choose the lower. Consider each defect-free configuration for $N_{T}$ as a stack of rows of bubbles. Then add (or subtract if $N<N_{T}$ ) bubbles to/from the middle of successive rows, starting from the row along the base of the triangle, until the number of bubbles reaches $N$. Then re-converge to equilibrium, allowing neighbour switching events on short edges where required.

\subsection{Square boundary}

Our candidate configurations are shown in figures 1 to 5 and the perimeters plotted in figure 6 and tabulated in Table 1. We confirm Tomonaga [22] and Bleicher's [23] conjectures for $N \leqslant 5$. 
The magic numbers are squares, of the form $N_{S}=i^{2}$ : although the number of defects is not minimized in these candidates, the defects lie along opposite edges of the square to leave hexagonal bubbles in the bulk. Figure 6(b) suggests that this does not result in a significant lowering of the perimeter compared to clusters for similar $N$.

Keeping the defects close to a pair of opposite boundaries is seen in many of the candidates. Again, it often appears to beat candidates that have few defects. As $N$ increases, an isolated 5-sided bubble in the bulk no longer generates a large penalty and is seen occasionally. About half of the candidates show reflective or rotational symmetry.

Following the derivation of (8), the square values of $N$ suggest the following expression for the number of peripheral bubbles:

$$
N_{p}=[4(\sqrt{N}-1)] .
$$

Were it not for the values $N=8,15$ and 23 , this would provide an upper bound, and it overestimates $N_{p}$ for the majority of the candidates.

A fit of the perimeters to the form in (1) gives $k=3.809$, less than in the triangular case but still significantly above the free case, reflecting the effect of confinement.

\subsection{Pentagonal boundary}

Our candidate configurations are shown in figures 1 to 5 and the perimeters plotted in figure 6 and tabulated in Table 1.

Five-fold rotational symmetry is exhibited for the pentagon for the magic numbers $N=6,16,31$, as for the circle [16], and may continue to be found for $N$ of the form $1+5 i(i-1) / 2$. In addition, the pentagon shows the same topology as the circle for $N=15,32,33$.

In general, however, the candidate configurations in a pentagon are highly disordered. With the further exception of $N=9,11,13,18,21,24$ and 34, all candidates for $N>7$ show at least one negative defect, usually in the form of a defect pair.

Based on the patterns for the magic numbers, we propose that the number of peripheral bubbles follows

$$
N_{p}=\left[\frac{5}{2}\left(\sqrt{\frac{8 N-3}{5}}-1\right)\right],
$$

which turns out provides a (tight) lower bound to the data.

A fit of the perimeters to the form in (1) gives $k=3.569$, slightly lower than the square case.

\subsection{Hexagonal boundary}

Our candidate configurations are shown in figures 1 to 5 and the perimeters plotted in figure 6 and tabulated in Table 1 . 
Many of these configurations are similar to either or both the circular and/or free cases $(N=1-12,14,17-22,24,25,27,29,30,34,35,37-39,41)$, including the magic numbers of the form $1+3 i(i+1)$.

There is a similar progression to that seen in the triangular case (e.g. for $30 \leqslant N \leqslant 42$ ) with a single defect pair that moves away from the boundary as $N$ increases. For lower $N$ this is disrupted by defect-free cases, that differ from the magic numbers by an uneven spacing of bubbles around the sides of the hexagon.

The number of peripheral bubbles is well-approximated by [14]:

$$
N_{p}=\left[3\left(\sqrt{\frac{4 N-1}{3}}-1\right)\right],
$$

which gets all but eleven values correct. The same expression gets all but thirteen values correct for the circular case and all but four values correct for free clusters.

The expression for the perimeter derived in (9), with $N_{p}$ given by (12) and $s=6$, works well here, indicating that the bubbles are quite regular. (This expression fails to describe the square and pentagonal data.) A fit of the perimeters to the form in (1) gives $k=3.452$, only slightly greater than in the circular case $(k=3.378[16])$ and the free case. In common with these two cases and the triangle, the partitions of the hexagon never show more than one negative defect.

\subsection{Discussion}

To test the potentials, we consider the circular case for which good candidate configurations exist [16]. No choice of potential finds all these candidates, but between the potentials all known solutions are found and one better solution for $N=40$ (both log and coulomb potentials). The coulomb potential performs best, finding 40 out of 42 of the known least perimeter configurations. For $N>25$ the least perimeter configuration rarely corresponds to the minimum energy arrangement of particles; rather, it is one of the local minima that gives it. In a small number of cases the least perimeter configuration is only found after performing T1s. In summary, there appears to be no "magic" potential for which the groundstate corresponds to the groundstate for the bubble cluster, and an optimal strategy should probably include variation of the potential as well as the random initial placement of the seed points.

How do the candidates compare for each $N$ ? Cox [16] showed that the least-perimeter partition of the circle is often the same as the solution in the free case, and it is also the case that the topology of the partitions of the hexagon are often the same. As the number of sides of the polygonal boundary increases, we expect this correspondence to be retained.

Referring to figure 1 , it is clear that for $N \leqslant 4$ the same topology solves all least perimeter partitions. Omitting the triangular case, which satisfies the procedure remarked upon above, the same topology solves the remaining five cases for $N=6$ and 7 , and omitting both the square and triangle, we find the same topology for $N=5,8,9,11,17$ and 18 . 
The positions of $s$ positive defects are pre-determined to lie in the corners of a polygonal boundary with $s$ sides, and this constrains the problem further. But for polygons with more than six sides (not considered here) there will be more defects than necessary, implying that a negative defect must always be introduced somewhere. A single sevensided bubble at the centre of the cluster and seven-fold symmetry overall, which was observed for the circle for $N=8$ and 22 (but not for $N=43$, the next in the sequence) [16] may be found for clusters confined within a regular heptagon, where the cost may be sufficiently low at higher $N$ for this topology to win.

The number $N_{p}$ of peripheral bubbles does not increase monotonically, although in all cases it scales roughly as $\sqrt{N}$ [14]. Despite the relatively high energy of the clusters in a pentagon, $N_{p}$ is lowest in this case. It is highest for the triangular clusters, because of the sharp corners.

A comparison of the perimeters of the candidates to each minimal perimeter problem is given in figure 6(a). It shows, for each $N$, the perimeter of the candidates for a free cluster and a cluster confined in a circular, triangular, square, pentagonal or hexagonal boundary. The perimeter increases monotonically with $N$ in all cases. It is clear that the lack of confinement in the free case leads to the lowest perimeter. Hexagonal clusters have slightly higher perimeter than those constrained by a circle, but the two are very similar. The triangular clusters have particularly high perimeter, followed by the square and then the pentagon.

We subtract the fit to the perimeter of the free clusters, $E_{f}(N) / L=3 N+3.068 \sqrt{N}$, from each set of data, shown in figure 6(b). It is now easier to see the "magic" clusters the dips in the data - where the perimeter is particularly low. These occur at the same $N$ for hexagonal, circular and free clusters.

\section{Least perimeter partition of the surface of a sphere}

We seek the least perimeter partition of the sphere into $N$ bubbles of equal area [30,31], equivalent to the energetic groundstate for $N$ monodisperse bubbles or the optimal packing of equal-area objects. We examine values of $N$ up to 32 and record the least perimeter and the configuration that realizes it.

Candidate configurations are shown in figure 7 , and the perimeters are tabulated in Table 2. In figure 8 the perimeters are shown and compared with a tiling of the plane with $N$ hexagons of area $A_{b}=4 \pi R^{2} / N$, which has $E_{\text {hex }} / L=3 N$ and therefore $E_{\text {hex }} / R=\sqrt{8 \sqrt{3} \pi N}$.

For $N=2-4,12$ we find the candidates for which proofs exist. For other $N \leqslant 10$, the candidates have the topology of the geodesic networks described in [28], with edges meeting at $120^{\circ}$. For example, the topology of $N=5$ consists of a pair of triangles covering the poles joined by 3 quadrilaterals, $N=6$ is cubic, $N=7$ consists of a pair of pentagons covering the poles joined by 5 quadrilaterals, $N=8$ consists of a pair of quadrilaterals covering each pole joined by four pentagons, and $N=10$ has quadrilaterals at the poles and two rows of four pentagons. 
A hexagon first appears in the case $N=11$ and the angles are no longer $120^{\circ}$. For $N=13$ it is not possible to insert just one hexagon and this is the highest $N$ for which a quadrilateral bubble appears; in fact, it has the same topology as the Matzke cell, one of the most common types of bubble in 3D monodisperse foams $[41,42]$.

For $N \geqslant 14$ it is apparent that all candidates found consist of 12 pentagons and $N-12$ hexagons. These are fullerenes, now well known from carbon chemistry. For example, the optimal candidate for $N=32$ is the $C_{60}$ fullerene, in which each pentagon is separated from the other pentagons by hexagons. Thus for $N \geqslant 14$ we conjecture that the best candidate can be found by finding the optimal location of the 12 pentagons in a partition that otherwise consists of hexagons.

\section{Conclusions}

We have found candidates to the minimal perimeter of partitions of a regular polygon with up to six sides into $N$ regions of equal area. Equivalently, we have found the global energetic groundstate of a monodisperse two-dimensional foam confined within a polygonal boundary. For the triangle we conjecture that optimal partitions for other $N$ can be found from the nearest "magic" cluster, i.e. for $N$ a triangular number, by adding/subtracting bubbles in successive layers from the wall. A similar procedure finds many candidates for the hexagonal boundary but not for any other boundary shape.

Few general results emerge from the data for square and pentagonal boundaries. Only defects with charge \pm 1 are observed (for $N>3$ ) and they tend to be close to the boundaries. Seven-sided bubbles are usually paired with five-sided bubbles.

We have also found candidates to the minimal perimeter of partitions of a sphere into $N$ regions of equal area. Equivalently, we have found the global energetic groundstate of a monodisperse two-dimensional foam confined to the surface of a sphere. For $N \geqslant 14$ all candidates are fullerenes. Thus, we conjecture that finding the least perimeter partition of the sphere for large $N$ is equivalent to the problem of finding the fullerene with the largest spacing between pentagonal faces.

For each $N$, the algorithm, even though repeated many times, explores only a few hundred different candidates at most. It remains an open question as to how many candidates actually exist, and whether it is possible to enumerate and test them in a reasonable time. Certainly, to extend the results presented here to higher $N$ will require an improved algorithm. Similarly, relaxing the condition of monodispersity, to consider for example bidisperse clusters [9], leads to many more candidates and the likelihood of fully exploring the space of all good candidates decreases.

\section{Acknowledgements}

We are grateful to E. Baumann, D. Ferguson, T. McDonough, F. Morgan, A. Mughal, E. Newkirk, B. Shuttleworth and W. Smith for useful discussions. SJC thanks K. Brakke 
for developing and maintaining the Surface Evolver software, and in particular for implementing the spherical arc mode used here, and R. Gabbrielli for introducing him to CaGe. SJC acknowledges funding from EPSRC (EP/D071127/1).

\section{References}

[1] D. Weaire and S. Hutzler. The Physics of Foams. Clarendon Press, Oxford, 1999.

[2] J.A.F. Plateau. Statique Expérimentale et Théorique des Liquides Soumis aux Seules Forces Moléculaires. Gauthier-Villars, Paris, 1873.

[3] J.E. Taylor. The structure of singularities in soap-bubble-like and soap-film-like minimal surfaces. Ann. Math., 103:489-539, 1976.

[4] W. Thomson. On the Division of Space with Minimum Partitional Area. Phil. Mag., 24:503-514, 1887.

[5] D. Weaire, editor. The Kelvin Problem. Taylor \& Francis, London, 1994.

[6] D. Weaire and R. Phelan. A counter-example to Kelvin's conjecture on minimal surfaces. Phil. Mag. Lett., 69:107-110, 1994.

[7] T.C. Hales. The honeycomb conjecture. Discrete Comput. Geom., 25:1-22, 2001.

[8] F. Morgan. Geometric Measure Theory: A Beginner's Guide. Academic Press, San Diego, 4th edition, 2009.

[9] M.F. Vaz, S.J. Cox, and M.D. Alonso. Minimum energy configurations of small bidisperse bubble clusters. J. Phys.: Condens. Matter, 16:4165-4175, 2004.

[10] M. Alfaro, J. Brock, J. Foisy, N. Hodges, and J. Zimba. Compound soap bubbles in the plane, SMALL Geometry Group report, 1990.

[11] J. Foisy, M. Alfaro, J. Brock, N. Hodges, and J. Zimba. The standard double soap bubble in R2 uniquely minimizes perimeter. Pac. J. Math., 159:3542-3546, 1993.

[12] F. Morgan. Mathematicians, including undergraduates, look at soap bubbles. Amer. Math. Monthly, 101:343-351, 1994.

[13] W. Wichiramala. Proof of the planar triple bubble conjecture. J. reine. angew. Math., 567:1-50, 2004.

[14] S.J. Cox, F. Graner, M.F. Vaz, C. Monnereau-Pittet, and N. Pittet. Minimal perimeter for $\mathrm{N}$ identical bubbles in two dimensions: calculations and simulations. Phil. Mag., 83:1393-1406, 2003.

[15] D. Weaire and N. Rivier. Soap, cells and statistics-random patterns in two dimensions. Contemp. Phys., 25:59-99, 1984. 
[16] S.J. Cox. Calculations of the minimal perimeter for $\mathrm{N}$ deformable cells of equal area confined in a circle. Phil. Mag. Letts., 86:569-578, 2006.

[17] F. Graner, Y. Jiang, E. Janiaud, and C. Flament. Equilibrium states and ground state of two-dimensional fluid foams. Phys. Rev. E, 63:011402, 2001.

[18] S.J. Cox and F. Graner. Large two-dimensional clusters of equal-area bubbles. Phil. Mag., 83:2573-2584, 2003.

[19] F. Morgan. Soap Bubble Clusters. Rev. Modern Phys., 79:821-827, 2007.

[20] A. Heppes and F. Morgan. Planar Clusters and Perimeter Bounds. Phil. Mag., 85: 1333-1345, 2005.

[21] A. Cañete and M. Ritore. Least-perimeter partitions of the disk into three regions of given areas. Indiana Univ. Math. J., 53:883-904, 2004.

[22] Y. Tomonaga. Geometry of Length and Area. Dept. of Mathematics, Utsunomiya University, Japan, 1974.

[23] M.N. Bleicher. Isoperimetric divisions into several cells with natural boundary. In Intuitive geometry. North-Holland, Amsterdam., 1987. Colloq. Math. Soc. János Bolyai, 48:63-84.

[24] E. Baumann, 2007. http://private.mcnet.ch/baumann/EqAreaOverview.htm.

[25] J. Masters. The perimeter-minimizing enclosure of two areas in $S^{2}$. Real Analysis Exchange, 22:645-654, 1996/7.

[26] M. Engelstein. The Least-Perimeter Partition of a Sphere into Four Equal Areas. Discrete Comput. Geom. (to appear). DOI: 10.1007/s00454-009-9197-8, 2009.

[27] T.C. Hales. The honeycomb conjecture on the sphere. arXiv:math/0211234, 2002.

[28] F.J. Almgren and J.E. Taylor. Geometry of soap films. Sci. Am., 235:82-93, 1976.

[29] B. Shuttleworth. Private communication, 2007.

[30] M. Goldberg. The Isoperimetric Problem for Polyhedra. Tohoku Math. J., 40:226236, 1934.

[31] E. Newkirk. Least-Perimeter Partitions of the Sphere. Undergraduate thesis, Williams College, Williamstown, Massachusetts, 2009.

[32] T. Hayashi and R.W. Carthew. Surface mechanics mediate pattern formation in the developing retina. Nature, 431:647-652, 2004.

[33] T. Tarnai. Optimum packing of circles in a circle. In I. Hargittai and T. Laurent, editors, Symmetry 2000, pages 121-132. Portland, 2002. 
[34] T. Tarnai and K. Miyazaki. Circle Packings and the Sacred Lotus. Leonardo, 36: 145-150, 2003.

[35] C.B. Barber, D.P. Dobkin, and H.T. Huhdanpaa. The Quickhull algorithm for convex hulls. ACM Trans. on Mathematical Software, 22:469-483, 1996.

[36] K. Brakke. The Surface Evolver. Exp. Math., 1:141-165, 1992. www. susqu. edu/brakke/.

[37] Y.-J. Lai and L. I. Packings and defects of strongly coupled two-dimensional Coulomb clusters:Numerical simulation. Phys. Rev E, 60:4743-4753, 1999.

[38] G. Brinkmann, O. Delgado Friedrichs, A. Dress, and T. Harmuth. CaGe - a virtual environment for studying some special classes of large molecules. MATCH Commun. Math. Comput. Chem., 36:233-237, 1997. http://www.mathematik.unibielefeld.de/ CaGe/.

[39] D. Ferguson. Private Communication, 2009.

[40] B.A. Shuttleworth. Isoperimetric problems in a triangle. Master's thesis, Aberystwyth University, 2008.

[41] E.B. Matzke. The three-dimensional shape of bubbles in foam - an analysis of the rôle of surface forces in three-dimensional cell shape determination. Am. J. Botany, 33:58-80, 1946.

[42] A.M. Kraynik, D.A. Reinelt, and F. van Swol. The structure of random monodisperse foam. Phys. Rev. E, 67:031403, 2003. 


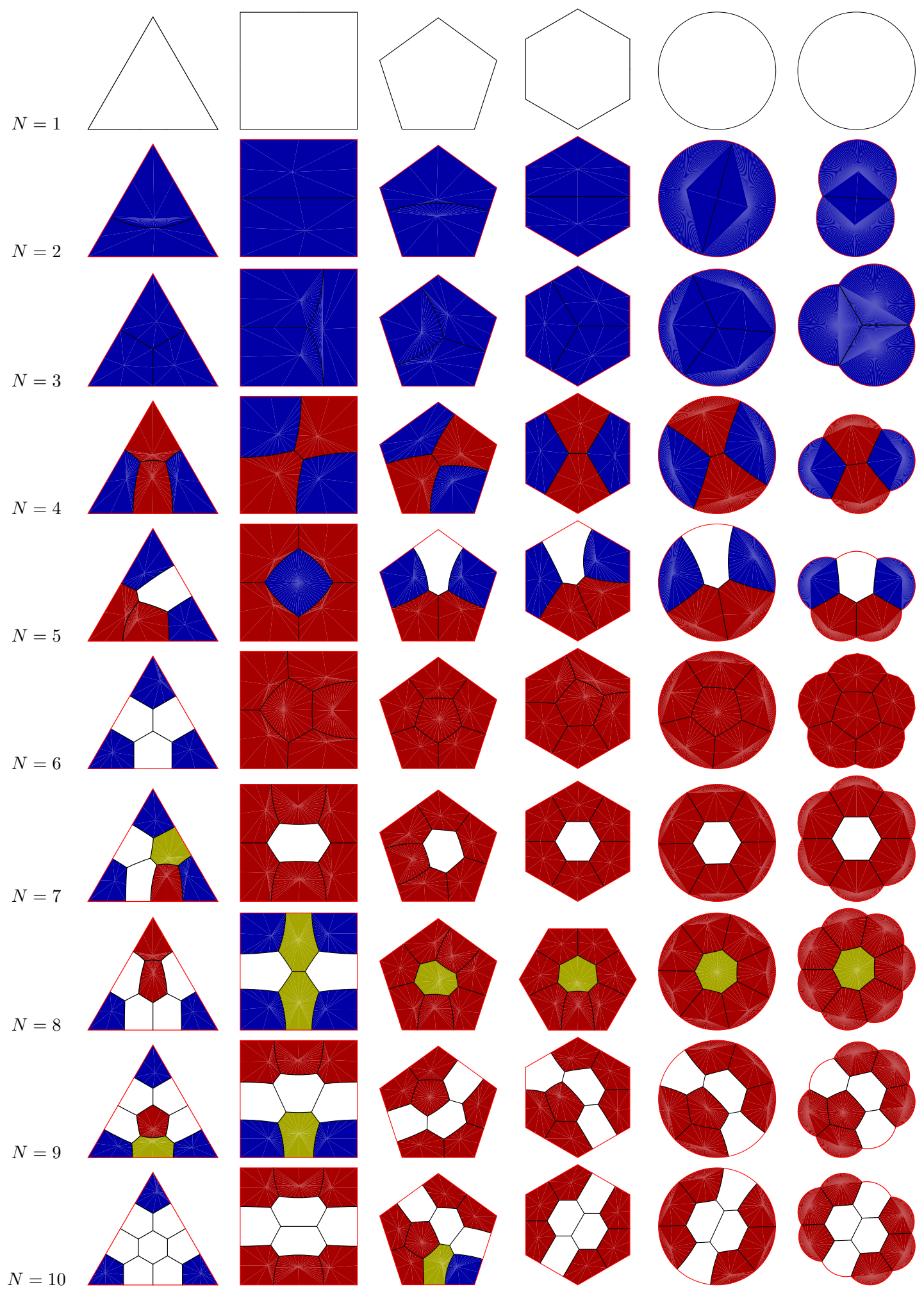

Figure 1: Least perimeter candidates for $N=1$ to 10 . 


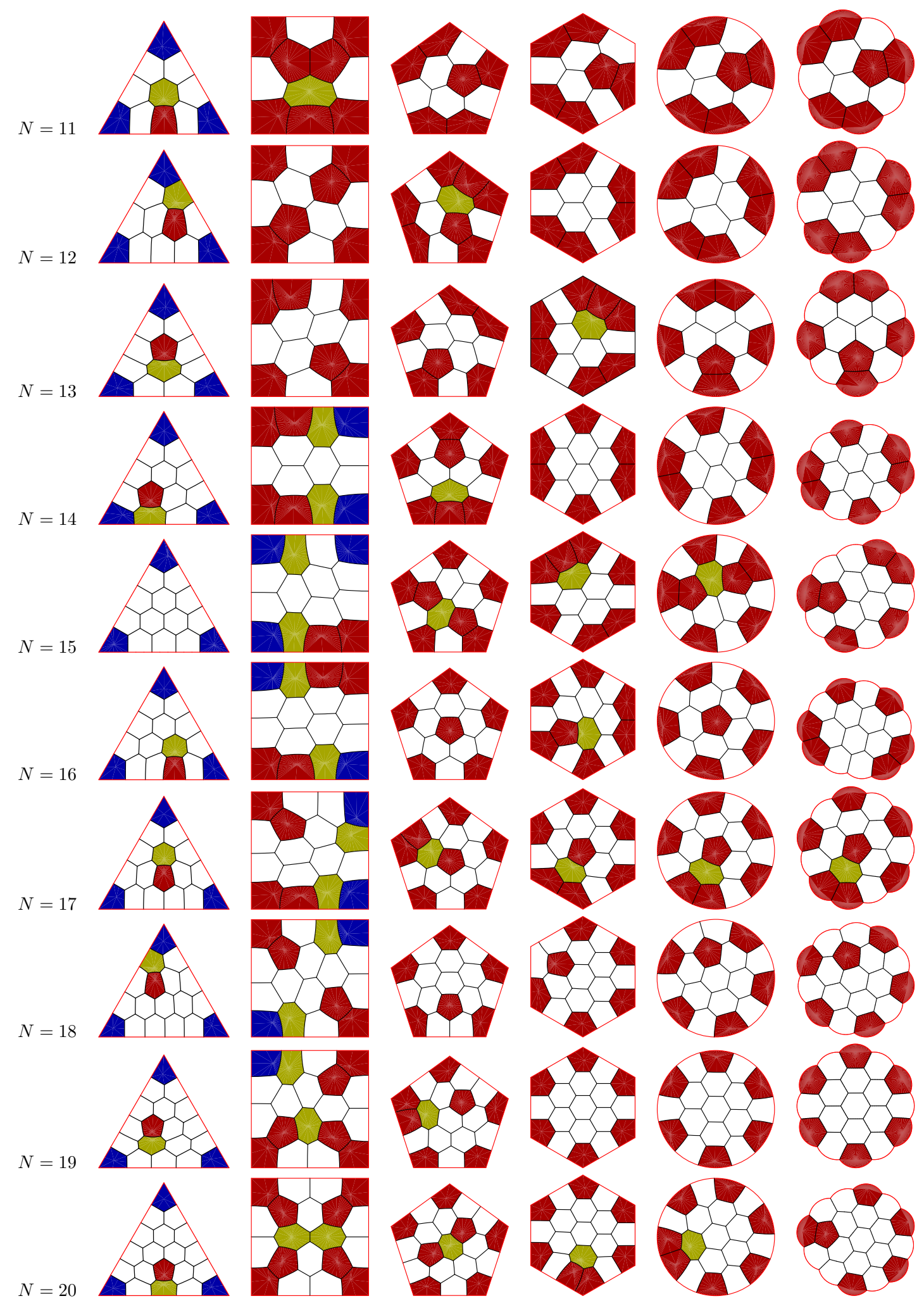

Figure 2: Least perimeter candidates for $N=11$ to 20 . 


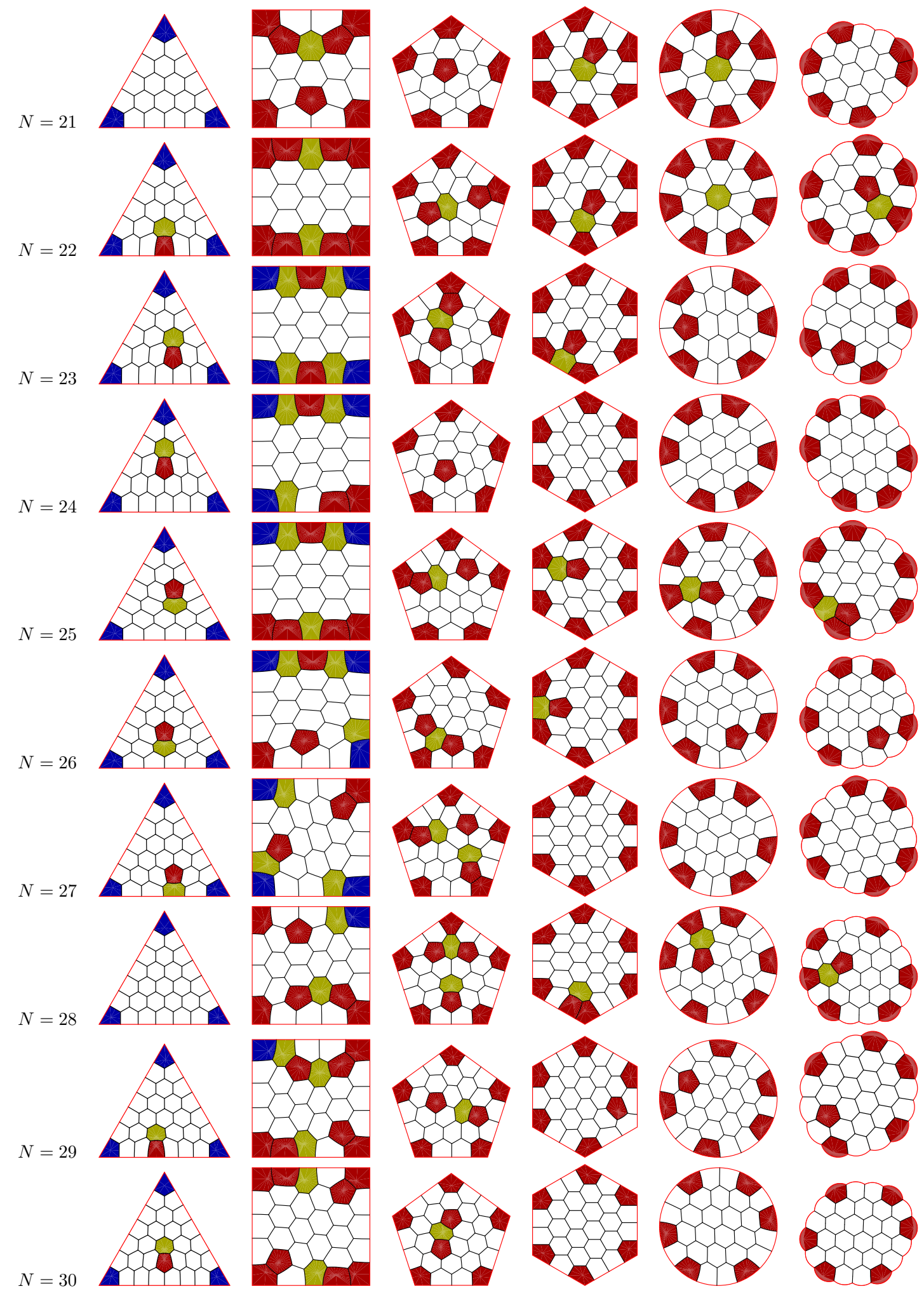

Figure 3: Least perimeter candidates for $N=21$ to 30. 


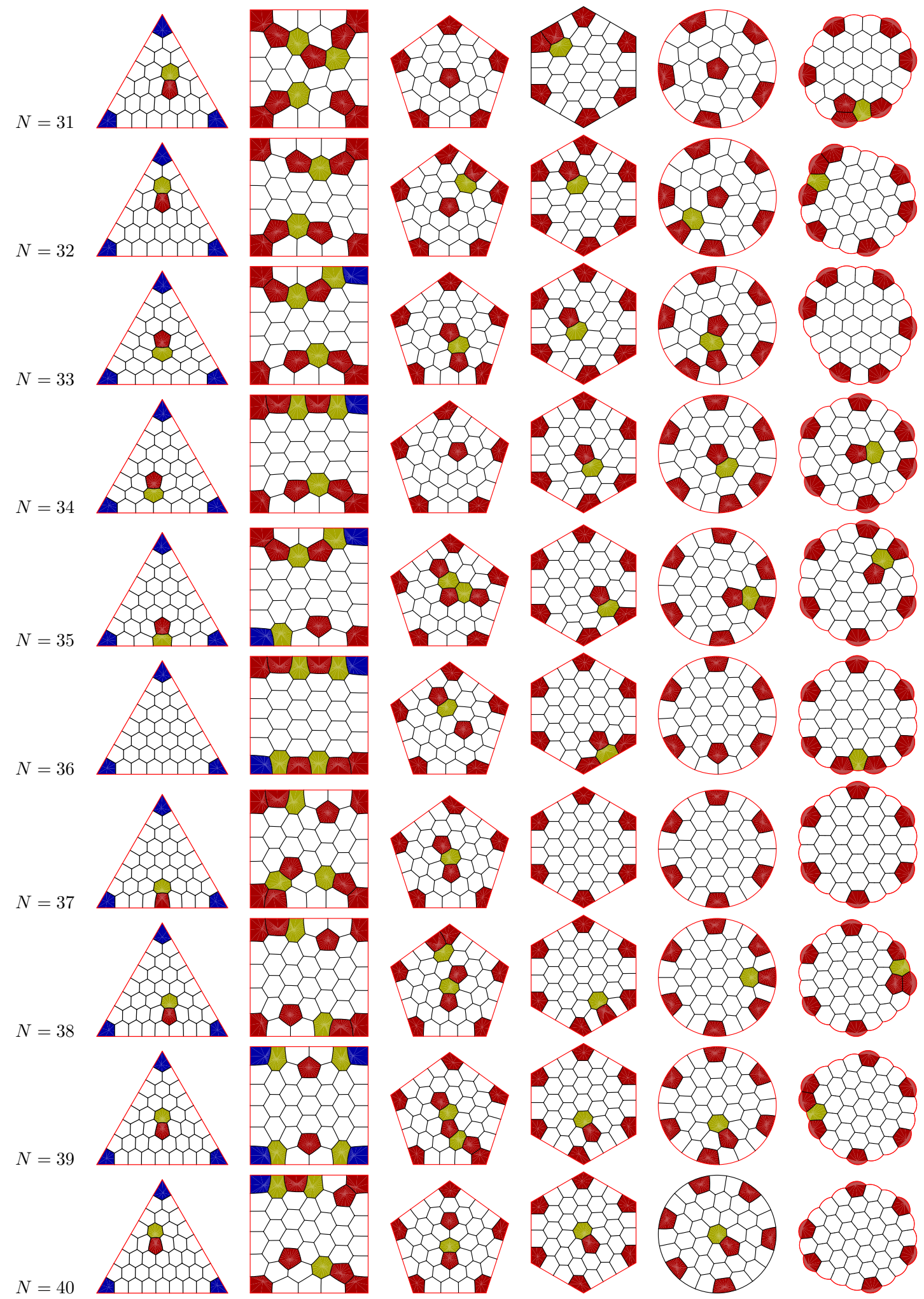

Figure 4: Least perimeter candidates for $N=31$ to 40 . 


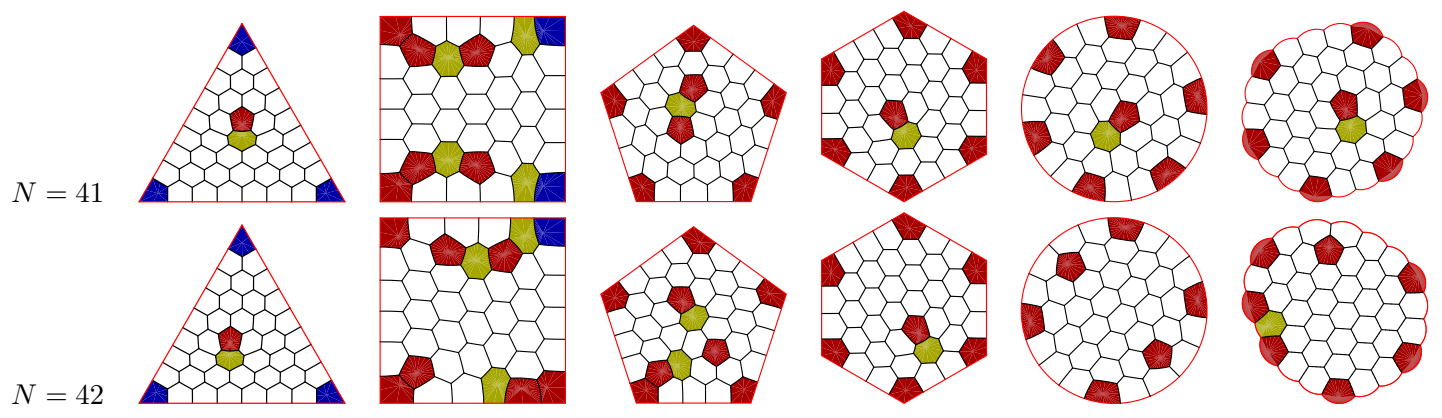

Figure 5: Least perimeter candidates for $N=41$ and 42 . 


\begin{tabular}{|c|c|c|c|c|c|c|}
\hline$N$ & triangular & square & pentagonal & hexagonal & circular & free \\
\hline 1 & 4.559 & 4.000 & 3.812 & 3.722 & 3.545 & 3.545 \\
\hline 2 & 7.895 & 7.071 & 6.934 & 6.784 & 6.609 & 6.359 \\
\hline 3 & 10.176 & 740 & 9.453 & 9.239 & 9.072 & 8.794 \\
\hline 4 & 13.085 & .951 & 11.945 & 11.743 & 11.542 & 11.188 \\
\hline 5 & 15.579 & 1.539 & 14.215 & 14.302 & 14.025 & 13.558 \\
\hline 6 & 17.615 & 16.999 & 16.325 & 16.476 & 16.155 & 15.777 \\
\hline 7 & 20.304 & .263 & 3.828 & 18.378 & 18.335 & 17.928 \\
\hline 8 & 22.759 & 21.490 & 21.183 & 20.955 & 20.634 & 20.200 \\
\hline 9 & 24.937 & 23.703 & 23.423 & 23.275 & 23.016 & 22.454 \\
\hline 10 & 26.902 & 26.025 & 25.684 & 25 . & 25.192 & 24.569 \\
\hline 11 & 29.455 & 28.286 & 27.830 & 27.684 & 27.361 & 26.773 \\
\hline 12 & 31.814 & 30.401 & 30.063 & 29.646 & 29.478 & 28.846 \\
\hline 13 & 34.046 & 32.716 & 32.210 & 32.043 & 31.706 & 31.049 \\
\hline 14 & 51 & 3 & 34 & 34 & 33.841 & 33.112 \\
\hline 15 & 38.046 & 37.058 & 36.313 & 36.365 & 36.041 & 35.313 \\
\hline 16 & 40.522 & 39.154 & 38.393 & 38. & 38.102 & 37.370 \\
\hline 17 & .794 & 1 & 1 & & 40.234 & 39.514 \\
\hline 18 & .043 & 43.511 & 42.873 & 42.559 & 42.282 & 41.554 \\
\hline 19 & 47.148 & 45.624 & 45.066 & 44. & 44.291 & 43.539 \\
\hline 20 & .192 & 47.6 & 13 & 46 & 44 & 45.738 \\
\hline 21 & 51.047 & 49.814 & 87 & 30 & 48.634 & 47.780 \\
\hline 22 & .467 & 51.991 & 51.402 & 51.070 & 50.759 & 49.921 \\
\hline 23 & 690 & 54.097 & 9 & 53. & 52.886 & 51.954 \\
\hline 24 & .884 & 56.2 & 70 & 86 & 54.928 & 53.930 \\
\hline 25 & .028 & 58.329 & 57.674 & 57. & 57.033 & 56.095 \\
\hline 26 & 62.069 & 60.576 & 59.722 & 59.361 & 59.078 & 58.108 \\
\hline 27 & 64.087 & 62.608 & 18 & 61 . & 61.077 & 60.067 \\
\hline 28 & 908 & 64.663 & 37 & 22 & 63.255 & 62.242 \\
\hline 29 & 68.289 & 66.675 & 65.774 & 65.617 & 65.301 & 64.245 \\
\hline 30 & .467 & 68.720 & 78 & 67.474 & 67.321 & 66.201 \\
\hline 31 & 72.631 & 70.775 & 47 & 69.777 & 69.462 & 68.364 \\
\hline 32 & 74.769 & 72.860 & 72.055 & 71.794 & 71.506 & 70.391 \\
\hline 33 & 76.824 & 74.986 & 74.131 & 73 . & 73.526 & 72.328 \\
\hline 34 & 78.842 & 77.078 & 47 & 75 . & 68 & 74.460 \\
\hline 35 & 80.832 & 79.220 & 78.297 & 77.809 & 77.557 & 76.456 \\
\hline 36 & 82.629 & 81.232 & 80.348 & 79.730 & 79.518 & 78.410 \\
\hline 37 & 84.980 & 83.390 & 82.391 & 81.470 & 81.486 & 80.344 \\
\hline 38 & 87.127 & 85.390 & & 83.798 & 83.669 & 82.507 \\
\hline 39 & 89.258 & 87.401 & 86.486 & 85.928 & 85.748 & 84.528 \\
\hline 40 & 91.377 & 89.496 & 88.529 & 88.011 & 87.791 & 86.465 \\
\hline 41 & 93.456 & 91.447 & 90.566 & 90.074 & 89.870 & 88.593 \\
\hline 42 & 95.469 & 93.545 & 92.618 & 92.108 & 91.892 & 90.608 \\
\hline
\end{tabular}

Table 1: Perimeter $E / L$ for the least perimeter candidates found here, normalized to bubbles of unit area. 


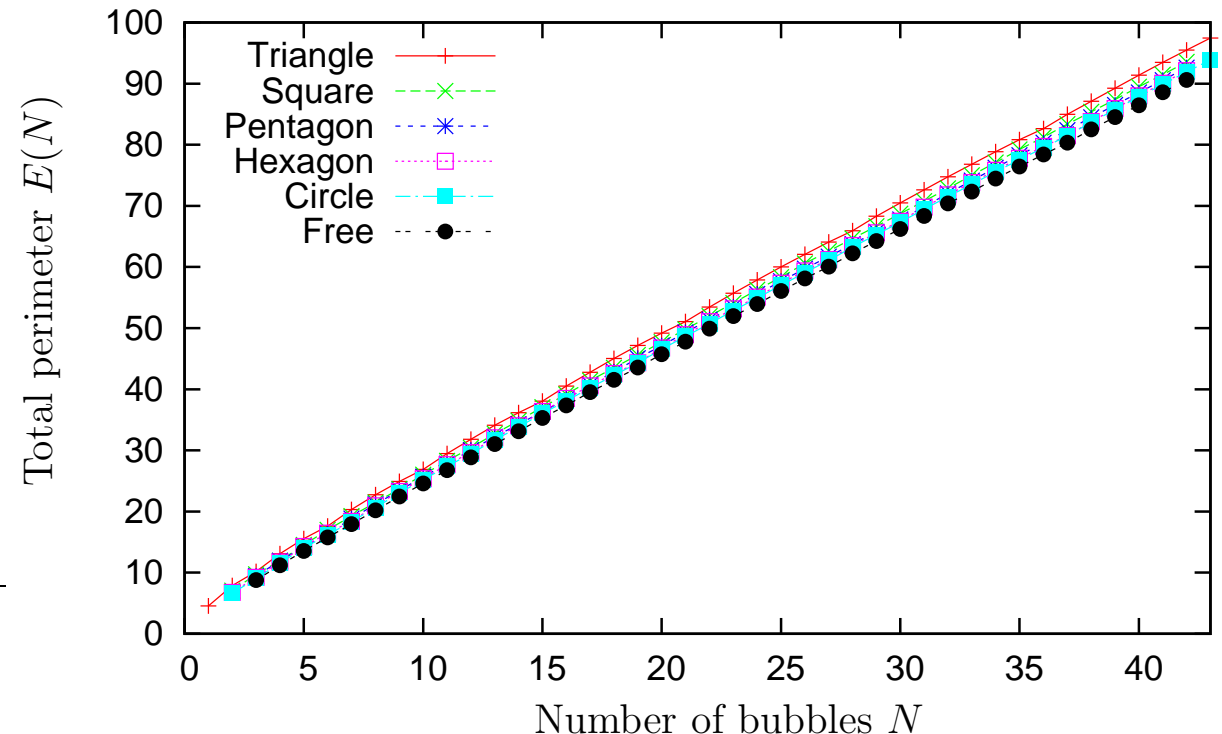

(a)

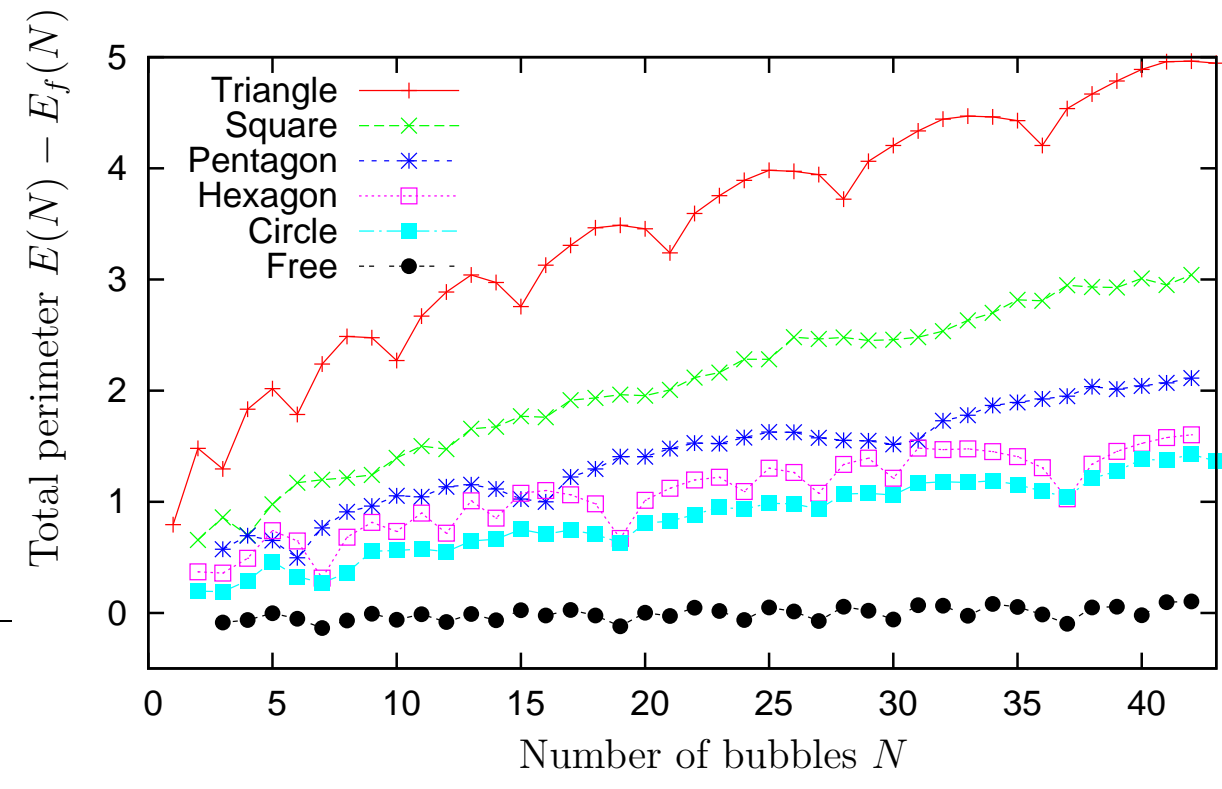

Figure 6: (a) Total perimeter of the least perimeter clusters found in the plane, normalized to bubbles of unit area. (b) Subtracting the fit (1) to the free clusters from each set of data separates the data and identifies the magic numbers. 


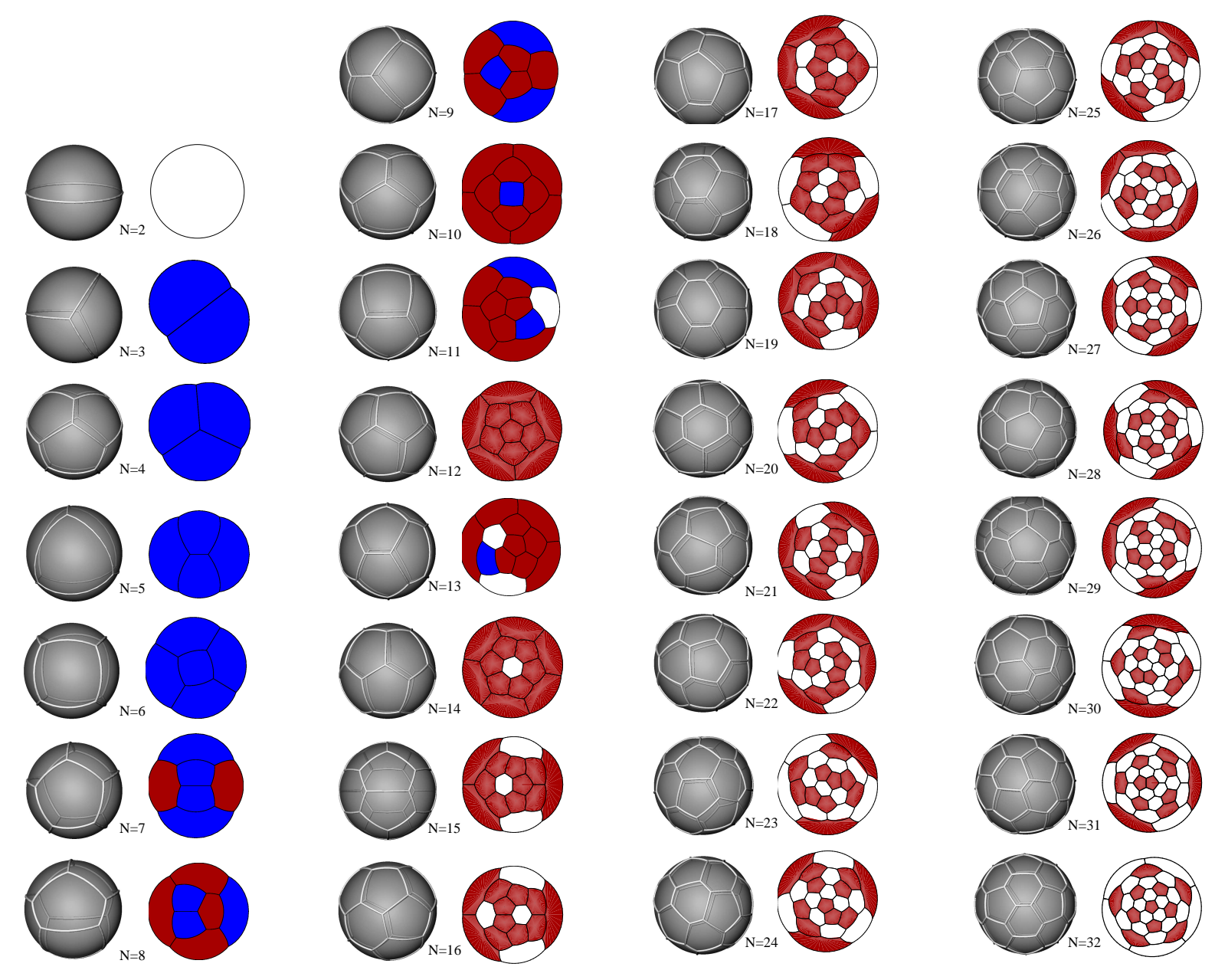

Figure 7: Candidate topologies for the least line length configuration of $N$ bubbles on the surface of the unit sphere, for $2 \leqslant N \leqslant 32$. In addition to showing the network of edges on the sphere, for each $N$ a representation based upon a Gauss map of the sphere surface to the plane (see §2) is given, with one bubble shown as the periphery of the cluster. 


\begin{tabular}{|c|c|c|}
\hline$N$ & $E / R$ & Topology \\
\hline 1 & 0.000 & - \\
2 & 6.283 & $1_{2}$ \\
3 & 9.425 & $2_{3}$ \\
4 & 11.464 & $3_{4}$ \\
5 & 13.452 & $3_{2} 4_{3}$ \\
6 & 14.772 & $4_{6}$ \\
7 & 16.360 & $4_{5} 5_{2}$ \\
8 & 17.710 & $4_{4} 5_{4}$ \\
9 & 18.867 & $4_{3} 5_{6}$ \\
10 & 20.015 & $4_{2} 5_{8}$ \\
11 & 21.163 & $4_{2} 5_{8} 6_{1}$ \\
12 & 21.892 & $5_{12}$ \\
13 & 23.112 & $4_{1} 5_{10} 6_{2}$ \\
14 & 23.964 & $5_{12} 6_{2}$ \\
15 & 24.891 & $5_{12} 6_{3}$ \\
16 & 25.736 & $5_{12} 6_{4}$ \\
\hline
\end{tabular}

\begin{tabular}{|c|c|c|}
\hline$N$ & $E / R$ & Topology \\
\hline 17 & 26.649 & $5_{12} 6_{5}$ \\
18 & 27.478 & $5_{12} 6_{6}$ \\
19 & 28.290 & $5_{12} 6_{7}$ \\
20 & 29.015 & $5_{12} 6_{8}$ \\
21 & 29.792 & $5_{12} 6_{9}$ \\
22 & 30.528 & $5_{12} 6_{10}$ \\
23 & 31.246 & $5_{12} 6_{11}$ \\
24 & 31.933 & $5_{12} 6_{12}$ \\
25 & 32.639 & $5_{12} 6_{13}$ \\
26 & 33.290 & $5_{12} 6_{14}$ \\
27 & 33.918 & $5_{12} 6_{15}$ \\
28 & 34.575 & $5_{12} 6_{16}$ \\
29 & 35.230 & $5_{12} 6_{17}$ \\
30 & 35.844 & $5_{12} 6_{18}$ \\
31 & 36.417 & $5_{12} 6_{19}$ \\
32 & 36.951 & $5_{12} 6_{20}$ \\
\hline
\end{tabular}

Table 2: Perimeter $E / R$ and topology of the candidates to the minimum perimeter partition into equal-area bubbles of the surface of the unit sphere. The topology is recorded in the form $a_{b}$, indicating $a b$-sided bubbles etc.

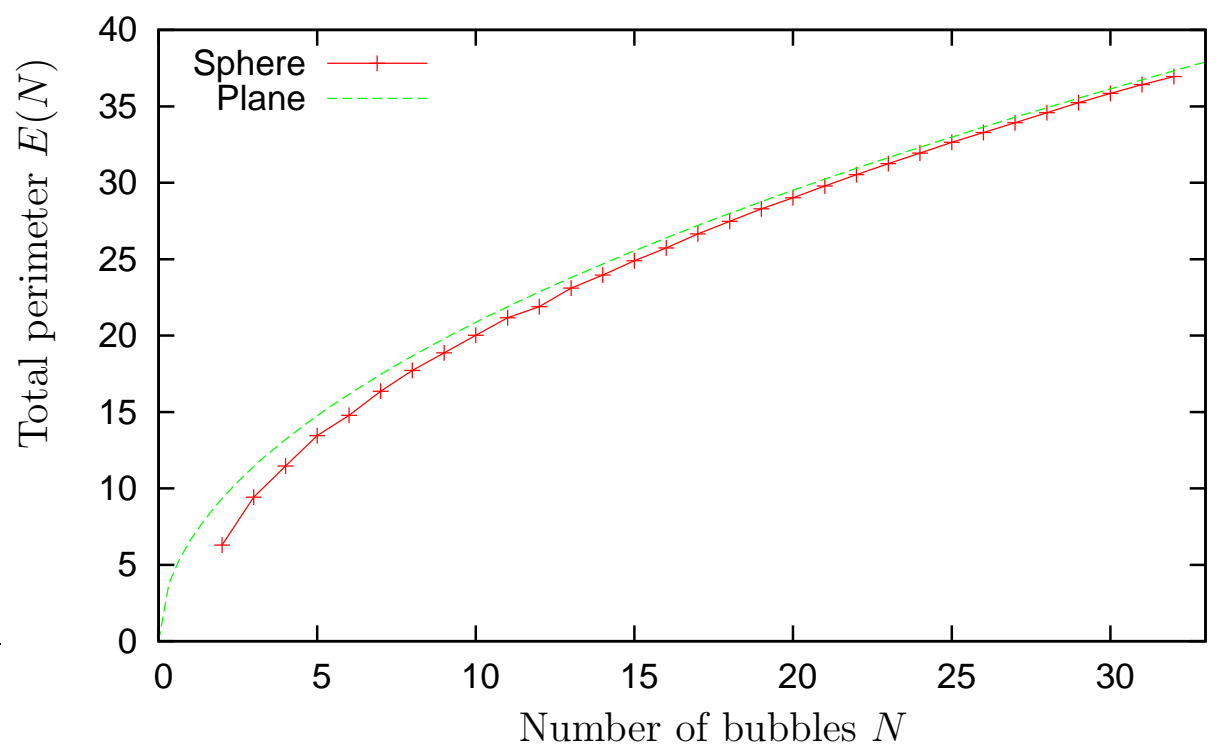

Figure 8: Total perimeter of the least perimeter tiling of the surface of the unit sphere with equal-area bubbles. Also shown is the energy of a hexagonal honeycomb with bubbles of the same area for each $N$, which is slightly greater. 


\section{Corrigendum - submitted Apr 27, 2010}

F. Morgan pointed out some errors in the data given for the least perimeter partition of the surface of the sphere into $N$ connected equal-area regions in our paper; we correct them here.

We consider the least perimeter partition of the surface of the unit sphere into $N$ regions of equal area. The edges should meet in threes at $120^{\circ}$, but each edge is not necessarily an arc of a great circle. The problem with our previous work was that the method used great circles, so that the $120^{\circ}$ condition could not be satisfied. We resolve this by adding another step to the numerical procedure: the best candidate for each $N$ is loaded back into Surface Evolver and the edges are subdivided into $2^{4}$ short segments. This structure is relaxed to a minimum of perimeter, meaning that the edges are no longer spherical arcs, but that they do meet at $120^{\circ}$; this reduces the perimeter by only a small amount (at most $2.5 \times 10^{-2}$ ). The perimeter $E$ and the pattern of this conjectured least perimeter solution are recorded in figure 9 . Note that the topology is the same as before in each case, but the perimeter has reduced slightly. Figure 9 also rectifies some small mistakes in the images of the spherical network of edges for $N=4,22,25$, although the projected images were correct. 

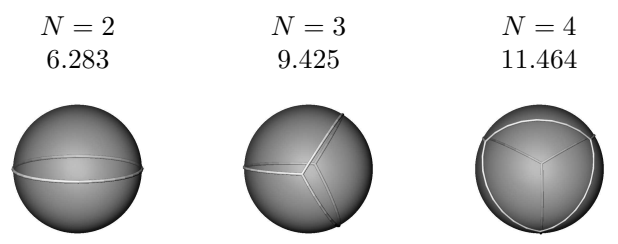

$N=5$
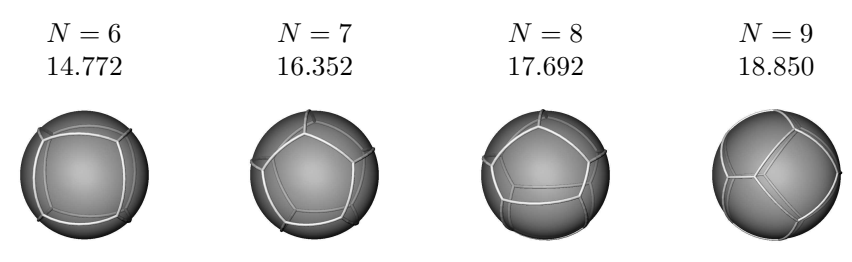

$N=10$

20.000

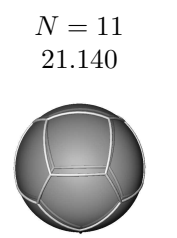

$$
\begin{aligned}
& N=12 \\
& 21.892
\end{aligned}
$$

$N=13$

23.095

$N=14$

$N=15$
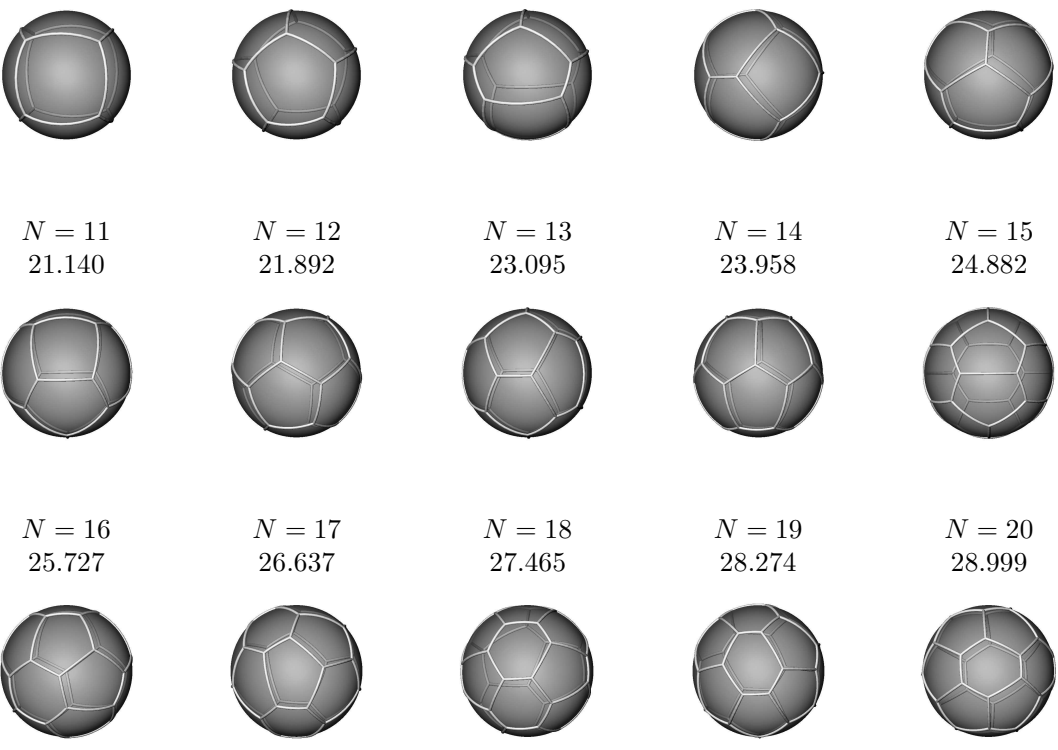

$$
\begin{aligned}
& N=17 \\
& 26.637
\end{aligned}
$$

$N=18$

27.465

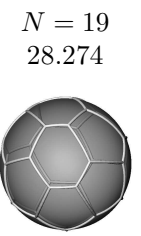

$N=20$
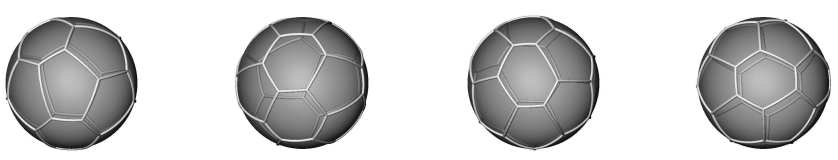

$$
N=21
$$

$N=22$

30.509

$N=23$

31.226

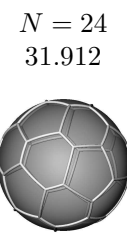

$N=25$

32.617
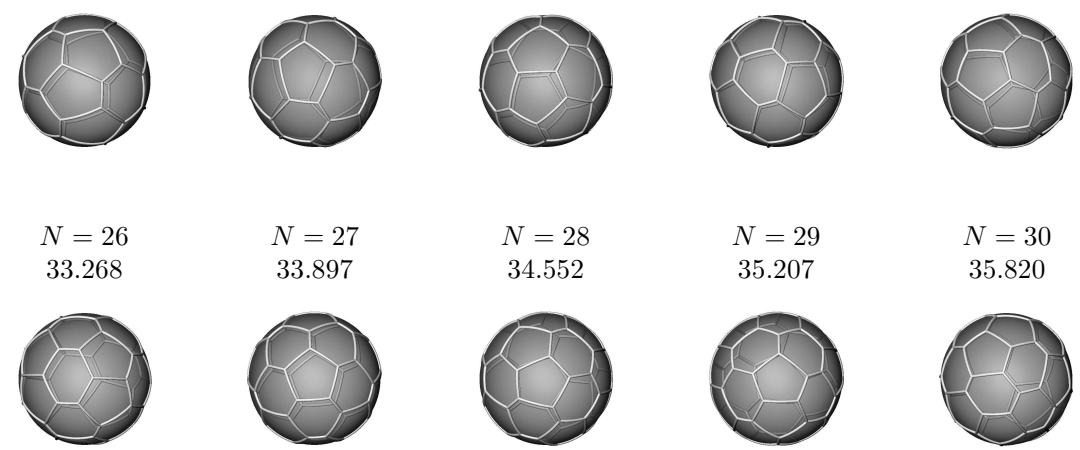

$$
N=27
$$

$N=28$

$N=29$

$N=30$

33.897

34.552

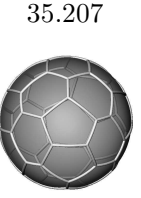

35.820
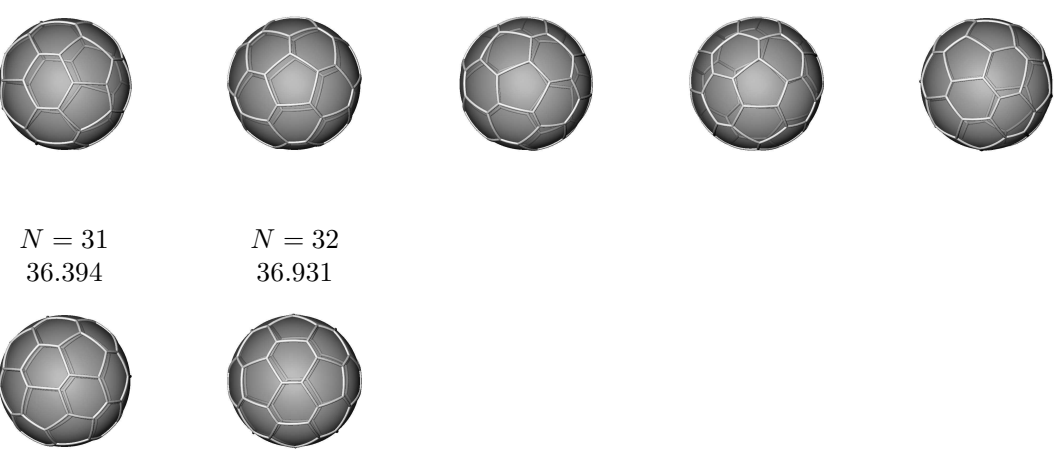

$N=32$

36.931

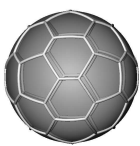

Figure 9: Candidate topologies for the least line length configuration of $N$ bubbles on the surface of the unit sphere, for $2 \leqslant N \leqslant 32$. 